\title{
Challenges and opportunities for informal physics learning in the COVID era
}

\author{
Michael B. Bennettఠ \\ JILA NSF Physics Frontier Center, 440 UCB, Boulder, Colorado USA 80309 \\ Department of Physics, University of Colorado Boulder, Boulder, Colorado USA 80309 \\ Kathleen A. Hinko and Dena Izadi® \\ Department of Physics and Astronomy, Michigan State University, East Lansing, Michigan USA 48823
}

(Received 5 March 2021; accepted 25 May 2021; published 13 July 2021)

\begin{abstract}
The COVID-19 pandemic has greatly impacted every aspect of academic activity, including the informal education and public engagement efforts of physics departments and institutions. As part of a large-scale national survey of the landscape of informal physics education programs, we have conducted a series of short interviews with directors and facilitators for a variety of programs in order to create a snapshot of whether and how programs have been able to adapt to the challenges and risks of the pandemic. Broadly, we find that programs are struggling to maintain any activity at all, and that those programs still in operation have relied largely on the efforts of individual director or facilitators to make substantial adaptations. Additionally, we find that those programs that have been most successful in maintaining activity benefit from strong, ongoing institutional support; we present the findings of this study both to complement existing research on formal spaces and to advocate for the continued support of informal physics education, which continues to play a crucial role in connecting departments to the public.
\end{abstract}

DOI: 10.1103/PhysRevPhysEducRes.17.023102

\section{INTRODUCTION}

The COVID-19 pandemic has deeply strained universities and colleges as they have struggled to adapt to the risks of contagion: classes adapted to remote facilitation; researchers precluded from collaborating in person; service projects and other extracurriculars in limbo due to shifts in institutional funding and tuition. Departmental public engagement is struggling as well, as communities and departments have prohibited in-person gatherings and enacted distancing protocols to keep participants safe. A tremendous amount of effort has, rightly, been expended to adapt some core departmental activities. Classroom and laboratory courses have been modified using a variety of techniques and methods, from fully remote instruction to hybrid techniques, to complete redesigns of course structures. Research over the last year has demonstrated impacts to student learning and attitudes, as well as to mental health [1-8]. As vaccines begin to roll out and departments begin to consider how to start building back to normal with the limited resources at their disposal, important questions loom: What activities to prioritize? And what activities to pause, ignore, or cease entirely?

Published by the American Physical Society under the terms of the Creative Commons Attribution 4.0 International license. Further distribution of this work must maintain attribution to the author(s) and the published article's title, journal citation, and DOI.
Naturally, formal classroom activity has been and should be a priority. But what of informal education efforts, those activities that promote learning outside of the classroom and often serve for departments as the primary means of interaction with the local community? In this work, we demonstrate that institutional support for informal education has been comparatively weaker than that for formal classroom activity and argue that it is in physics departments' best interest to treat public engagement and informal education as a core component of activity while considering how to recover from the pandemic.

\section{FRAMEWORK AND PRIOR RESEARCH}

In this work, we follow the National Research Council in defining "informal education" as that education which takes place outside of a formal classroom: in museums, after-school programs, science centers, etc. People of different ages, ethnicities, and socioeconomic statuses engage in informal education [9]; in science, technology, engineering, and mathematics (STEM), and particularly in physics, it has long been a hallmark for scientists to facilitate informal learning through public engagement or "outreach" programs. These programs often play a crucial role in connecting physics departments to their local community, and have many well-documented benefits to public participants [10-13]. They also provide opportunities for physics students to practice pedagogy and public speaking [14-19] and to find a sense of belonging in a 
physics community [20], which have implications for recruitment and addressing issues of representation [21-24]. Departmental public engagement efforts are often run by undergraduate and graduate volunteers, student groups, or by individual staff members; unfortunately, departmental priorities often relegate informal physics programs to the fringe of departmental activity despite these benefits. As we will demonstrate in this paper, the circumstances under which informal programs operated pre-COVID are likely untenable in the post-COVID era.

This work complements ongoing research on assessing the impact of COVID in the formal space, as well as builds on our ongoing work on the landscape of physics public engagement in the United States [25,26]. Using a framework from organizational theory, we have directed our investigation according to three major research questions:

1. What aspects of existing informal physics programs have been affected by COVID?

2. How have programs adapted to the limitations imposed by COVID?

3. How are programs looking toward the future?

To answer these questions, we have interviewed lead facilitators for a subset of informal physics education programs throughout the U.S. about their experiences during the pandemic. Using qualitative analysis, we have categorized ways in which these programs have responded to the unique challenges presented by the pandemic. Implications of our findings are crucial for understanding how to better support these programs and how to prepare for future operational uncertainties.

In our previous efforts in mapping the landscape of the informal physics efforts across the nation, we conceptualized the organization theory framework in order to characterize the nature of some (not all) informal physics activities facilitated by physics institutions (university departments, national labs, and research centers) [25-29]. For the purposes of this paper, a "program" is an organized entity with the expressed purpose of conducting public engagement and a history of implementing efforts or products to that end. We have operationalized six main categories of informal physics organizations that contribute to functionality: personnel (the physics students and physicists who run the programs), audience (youth and adult participants), program (content and format of the main activities), resources (funding, physical equipment, venues, etc.), institution (connections and support from the university or center), and assessment (impact measurement). This operationalized framework formed the basis for a new set of emergent codes reflecting program adaptations during the pandemic for this work, generated during the course of interview analysis.

\section{METHODS}

As mentioned above, this study was predicated in part upon data from a larger systematic survey of the national landscape of informal physics education programs [26]. Our goal for this project was to produce a snapshot of that landscape during COVID-our sample thus consisted largely of programs we could quickly gain access to. In August and September of 2020, we conducted and recorded interviews with 15 program leaders from 14 unique informal physics programs across the country using Zoom software. Program formats (established in prior work) included five "presentation" -style programs, such as public lectures, four "camp" -style programs, three "club" -style programs such as afterschool programs, and one "festival" -style program. Nine of these programs were previously involved in the broader landscape study; six were newly interviewed for this study. Interviews were transcribed using voice recognition software and spot checked by the researchers for accuracy. Prior to analysis, interviewees and programs were assigned pseudonyms to preserve anonymity.

In order to build a dataset on both programs' pre-COVID activity and their novel or adapted activity during the pandemic, we modified the original landscape study interview protocol, adding a section with pandemic-specific questions. Interviews were conducted with either the complete protocol or the small, COVID-only protocol depending upon whether the program had been interviewed before or not, in order to ensure that returning and new interviewees produced comparable data. Questions in the new protocol focused on adaptations to programming, personnel changes, etc. Interviews took approximately 30 and 90 min for returning and new subjects, respectively. Once the interview data were collected, we coded interviews using a set of COVID-specific labels created emergently from analysis of two of the interviews, one from a returning interviewee and one from a new interviewee. These COVID-specific codes were then applied across the rest of the interviews. Codes used are shown in Table I along with short descriptions. For reliability, two of the authors coded separate segments of the data from six

TABLE I. The five top-level codes we used in the analysis, along with a few representative subcodes from each category of top-level code. These codes were articulated emergently.

\begin{tabular}{lc}
\hline $\begin{array}{l}\text { Top-level } \\
\text { code }\end{array}$ & \multicolumn{1}{c}{ Example subcodes } \\
\hline Program & $\begin{array}{c}\text { Adapted in-person activities; adapted virtual } \\
\text { actvities; new program directions; concerns about } \\
\text { program continuity; new ideas for implementation }\end{array}$ \\
Personnel & $\begin{array}{c}\text { Recruitment or retention problems; facilitator's } \\
\text { future COVID outlook }\end{array}$ \\
Resources & $\begin{array}{c}\text { COVID impact on partners \& partnerships; venue } \\
\text { closures during pandemic; financial difficulties }\end{array}$ \\
Institution & $\begin{array}{c}\text { Changes in institutional support or priorities } \\
\text { Audience }\end{array}$ \\
$\begin{array}{c}\text { Participant risk; changes in the audience } \\
\text { participation; community response to COVID }\end{array}$ \\
\hline \hline
\end{tabular}


interviews and compared coding, discussing, and reassigning, as well as refining the definitions of the codes, until agreement was reached.

\section{FINDINGS}

As expected, our interviews revealed that almost all aspects of any program's operation were affected by the pandemic.

\section{A. Closure and related challenges}

At the time of the interviews, seven programs $-50 \%$ of our sample-had ceased operation entirely. An exchange between the interviewer (I) and Kevin (K), a faculty supervisor for a museum-partnership program, is representative of the struggles of many of the facilitators we interviewed:

I: And you can say anything else you want to, of course, I'd just like to hear how the program's operations have been impacted by the recent COVID-19 pandemic.

K: Yeah, I mean, it's a full stop. And it's, basically, when they [the museum] open up again, and they implement whatever procedures that they need, they will have to sort of figure it out, I guess.

Institutional concerns about safety-e.g. school district distancing policies or university restrictions-were the number one source of program closure. University restrictions in particular affected programs with broad audience ranges, not simply children. Even among those programs whose community partners were receiving volunteers, the logistical pressures of maintaining COVID-safe activity often resulted in the decision to cease in-person activity. Both operational and nonoperational programs reported significant financial struggles as well, whether due to budget or priority shifts at home institutions or lack of ticketing income. One program manager, Chelsea, described furloughs at her museum or university-supported program:

“... all of our student staff, all of our operating costs, our full time staff... are paid by [Chelsea's department]they're furloughed. I'm not $100 \%$ sure that I'm going to make it out of this with the job and everything. So I know that our annual operating budget has actually been cut by, I think it was, 5\% as it was, which is not something we've ever really had to deal with before. Anything that affects [Chelsea's department] affects our salaries and therefore that sort of thing we have, but not our personal operating budget because we get money through field trips and ticket sales. And we had to close in our busiest time. And so all of that money has gone. We aren't making any money this year. I am starting to charge for virtual field trips. And so that's bringing in some funding, but not nearly as much as we normally would have gotten."
Most public engagement is undertaken by faculty and students situated within departments and institutions; many interviewees reported that, due to COVID, they were scrambling to create online university courses, maintain activity in other professional areas, or simply trying to get by. Mark, a faculty advisor for a student-led program that performs physics demonstrations at local schools, described his experience: “... so [the program is] run with student leadership on a volunteer basis. And the leaders are dealing with their own stresses right now, having to deal with their classes and their housing... And that's something even-making the situation even worse... even if there were a serious plan to say, 'We're going to put together one really awesome Zoom science show,' right now, they don't have the time to work on it." Other challenges to programs'operations included lack of student volunteers due to shifts to remote learning, lost connections to community partners, closures of external venues, and inability to "faithfully" adapt program format to virtual activity, among others. Interviewees also expressed concerns about future activity and even the fate of their programs.

\section{B. Adapting programs in response to COVID}

The other seven programs in our sample were able to adapt some online activities for their audience, and one of those programs had also developed novel outdoor activities for small groups of students. As expected, given the progression of the pandemic, most programs had been unable to access physical venues since mid-to-late March 2020. As with the inactive programs, institutional concerns such as safety regulations prompted a great degree of uncertainty for adaptation. Programs also reported scrambling to meet the constraints of COVID restrictions without incurring a substantial lapse in audience engagement.

We observed a wide variety of adaptations to the COVID pandemic between programs, the most common being the transformation of in-person activities to virtual activities. Other examples include public science-on-tap talks adapted for Zoom; camp-style programs creating kits of equipment which were shipped to participants for remote facilitation; and even programs designing completely new curricula for maker-style museum efforts. Promisingly, we did not document any examples of programs making adaptations that failed - that is, every program that attempted adapting to the constraints of COVID was, at the time of analysis, still active.

Not all reported impacts of COVID were negative. A few programs reported positive programmatic changes likely to persist beyond the pandemic, most notably newly developed forms of participant or audience engagement. For example, Hillary, who is the lead facilitator of a campformat program with over 40 volunteers, reported that remote facilitation had enabled her program to engage students in other states: “...So that was something new that 
we hadn't really thought about. Before we were so kind of in our little bubble and focused on that community for so long, we didn't realize that our programming could go nationwide. So that's something that's been really exciting." Hillary also designed completely new protocols for maintaining social distancing, checking students in and out, and other safety issues in order to maintain in-person activity. She noted that her program had actually increased enrollment: “... I think if anything, we're probably busier than we were at this time last year and offering more classes; we're really trying to front-load this school year with as many outdoor classes as we can because of weather ... and then after that, we're kind of gonna focus on virtual again, or maybe one-day classes, we have some snow school curriculum... so we're looking at that possibly being an outdoor class." As we discuss below, this atypical success story is likely the result of unique institutional support.

\section{Future of program operations}

At the time of interviewing, most programs did not have a strong sense of when operation would return to its preCOVID "normal." Most facilitators reported skepticism about a return to traditional operation, even those that had planned in-person events. Some programs expressed hope of re-opening programs fully by 2021-however, as of February 2021, we are not aware of any programs that have returned to full operation, including those programs that explicitly planned for it. Programs' ambiguous outlooks on future operation resulted in a variety of hypothetical opening scenarios: opening up physical spaces for smaller groups of people while following state safety orders; special events for first responders, eventually opening to very small subsets of the public; waiting on partners and venues for opening decisions; holding events outdoors; utilizing surveys to get a sense of participant or audience interests and levels of concern; and others. Figure 1 summarizes the various outlooks reported by the programs we studied.

\section{Current and future status of the programs}

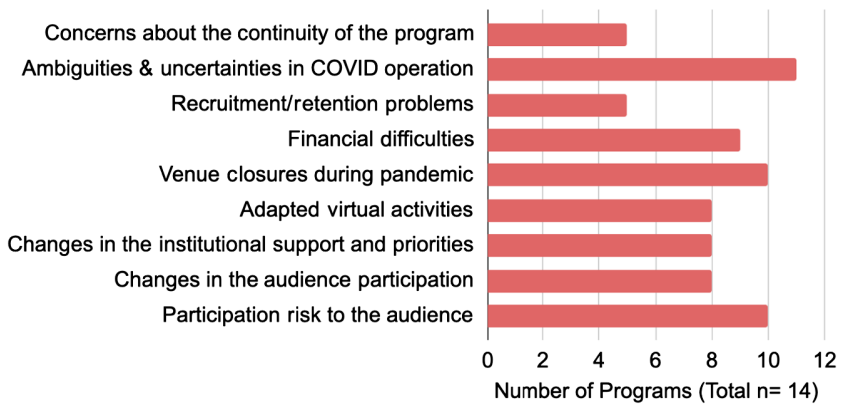

FIG. 1. Counts of programs in our sample whose lead facilitators reported concerns about their current and future status.

\section{DISCUSSION}

In dealing with the challenges and risks presented by program operation during COVID, a pertinent question is which factors have allowed some programs to thrive while others close their doors. Crucially, among our study population, those programs who have most successfully adapted have been those with institutional flexibility and strong, systemic support. Additionally, program directors were observed to have an outsized role in harnessing these resources, when they existed, to maintain or grow program activity, either leading efforts or enacting changes completely on their own, and, most notably, spearheading efforts to increase program capacity.

Hillary's program in particular stands out as an example of success, having actually increased its operation and having been the only program to have returned to in-person activity during the pandemic. Hillary's co-leader, Alice, who was also interviewed, described the program's positionality within its institution: "I would say, before COVID, in a general year, on average ... about $60 \%$ of our funding came from [admissions in] the summer program, about $20 \%$ came from grants and gifts ... and then another $20 \%$ from the Division of Continuing Education [at Alice and Hillary's large R1 University]. I would say, generally, it would be about 60/20/20. This year, obviously, our revenues were down for our summer programs, because we were able to offer a lot less. And so Continuing Ed helped to support some of that difference from what we had initially budgeted."

The implication here is striking, if not wholly unexpected: strong institutional support has not only played a crucial role in programs' ability to survive the pandemic, but has served as a foundation for programs to build novel systems for successful operation postpandemic. Ellen, an R1 faculty involved in a museum-based research-practice partnership program, described how, despite a full stop in museum admissions, support from an NSF grant had allowed her to maintain support for some of her activity: "So they're kind of down to a skeleton crew. Once [the museum] opens again, hopefully, they'll be able to rehire people. But right now, they're a skeleton crew. And we're paying them as much as we can out of the NSF grant."

Ellen also described how this support enabled her to continue activity and create program content that could be shared with new populations of students who would normally be out of range to visit her partner museum. Hillary and Alice similarly described how the lowerstimulation remote environment produced more positive outcomes in students with behavioral difficulties. Both programs hope to maintain these new developments postCOVID. Other programs described adaptations catalyzed by the pandemic as well, in some cases building on preCOVID interests in these innovations. Again, however, the most successful adaptations were those from programs with strong, centralized institutional support. 
In contrast, those programs without full integration into their institutions' organizational structure were most likely to report struggling or complete closure. These programs, like Kevin and Chelsea's above, were not even always certain that they would be able to reopen after COVID. Not only would this outcome represent a tremendous loss of immediate value to the public and the participants programs hope to serve, it also seriously undermines departments' efforts to enrich students. As mentioned above, informal programs improve students' ability to engage in science fluently in science communication, their perception of the purpose of public engagement, and even their content knowledge [14,24,30,31]. Participation in these programs is a crucial way for students to gain facility with pedagogical techniques; these efforts are, again, also often the primary way in which departments interact with the community. Like any relationship, departmental relationships with local communities must be maintained to survive. Additionally, programs frequently fill their ranks primarily through word-of-mouth recruitment among students [24]. Although it may be tempting, as departments assess how to recover from the turmoil of the pandemic, to deprioritize public engagement as ancillary activity, we exhort departmental leaders to consider the fact that, without ongoing support, programs risk dissolving entirely-and with them, our connection to the very populations we wish to serve through public engagement.

\section{CONCLUSIONS}

Unsurprisingly, and yet worth emphasizing again: strong institutional support is, as seen here, a crucial component of informal physics programs' ability to weather the storm of COVID and emerge from the pandemic poised to bounce back stronger than before. We therefore return to a question posed in Sec. I: How should departments prioritize resource allocations as they recover from this catastrophe?
Given the crucial role that informal physics programs play in training departmental students in pedagogy, shifting departmental culture, changing attitudes and beliefs about science and public speaking for both department members and external participants, and-most importantly - connecting departments to the communities they live and work alongside every day, we implore institutional leaders to consider the findings of this study as they decide how to restructure and support public engagement moving forward.

With some of the programs we interviewed having stopped activity for a year or more, many program directors are anxious about whether their departments and institutions will consent to funding activity in the future at all; subjects also reported anxiety about whether audiences would be lost entirely if programming could not keep up momentum. While we acknowledge that not every department or institution has the level of support of, e.g., an NSF-funded program, and are not advocating for supporting informal learning at the expense of other core departmental efforts in classrooms and labs, we urgently suggest that departments view their informal programs as exactly that-core elements of their mission. Without support, informal physics programs may not survive the pandemic, and with them would die one of the most important and historical ways that departmental scientists engage with the world they inhabit.

\section{ACKNOWLEDGMENTS}

The authors would like to gratefully acknowledge the participation of informal educators across the nation, especially during a stressful and busy time. This work is supported by the JILA NSF Physics Frontier Center (NSF PHY-1734006) and by NSF Grant No. DRL-1713060. Any opinions, findings, and conclusions or recommendations expressed in this material are those of the author(s) and do not necessarily reflect the views of the National Science Foundation.
[1] B. Wilcox and M. Vignal, Understanding the student experience with emergency remote teaching, in Proceedings of the 2020 Physics Education Research Conference, Virtual Conference (AIP, New York, 2020), pp. 581-586.

[2] A. Gavrin, Physics students' reactions to an abrupt shift in instruction during the COVID-19 pandemic, in Proceedings of the 2020 Physics Education Research Conference, Virtual Conference (AIP, New York, 2020), pp. 167-172.

[3] A. Olsho, S. W. Brahmia, C. Zimmerman, T. I. Smith, P. Eaton, and A. Boudreaux, Online administration of a reasoning inventory in development, in Proceedings of the 2020 Physics Education Research Conference, Virtual Conference (AIP, New York, 2020), pp. 376-381.
[4] M. Fields, B. Modir, W. G. Newton, R. Lock, and J. C. Stanfield, The transition to online teaching during the COVID-19 pandemic at a regional, rural university: The experience of learning assistants, in Proceedings of the 2020 Physics Education Research Conference, Virtual Conference (AIP, New York, 2020), pp. 149-154.

[5] M. W. Guthrie, T. Zhang, and Z. Chen, A tale of two guessing strategies: Interpreting the time students spend solving problems through online log data, in Proceedings of the 2020 Physics Education Research Conference, Virtual Conference (AIP, New York, 2020), pp. 185-190.

[6] G. Corsiglia, T. Garcia, B. P. Schermerhorn, G. Passante, H. Sadaghiani, and S. Pollock, Characterizing and 
monitoring student discomfort in upper-division quantum mechanics, in Proceedings of the 2020 Physics Education Research Conference, Virtual Conference (AIP, New York, 2020), pp. 92-97.

[7] M. F. J. Fox, A. Werth, J. R. Hoehn, and H. J. Lewandowski, Teaching labs during a pandemic: Lessons from Spring 2020 and an outlook for the future, arXiv:2007.01271.

[8] F. R. Bradbury and C. Pols, A pandemic-resilient openinquiry physical science lab course which leverages the maker movement, arXiv:2006.06881.

[9] P. Bell, B. Lewenstein, A. W. Shouse, M. A. Feder et al., Learning Science in Informal Environments: People, Places, and Pursuits (National Academies Press, Washington, DC, 2009).

[10] M. Phipps, Research trends and findings from a decade (1997-2007) of research on informal science education and free-choice science learning, Visitor Studies 13, 3 (2010).

[11] R. Dou, Z. Hazari, K. Dabney, G. Sonnert, and P. Sadler, Early informal stem experiences and stem identity: The importance of talking science, Sci. Educ. 103, 623 (2019).

[12] Z. Hazari, R. Dou, G. Sonnert, and P. Sadler, Breaking with tradition: How informal learning experiences in physics contribute to physics identity development (2019), https:// www.compadre.org/per/perc/2019/Detail.cfm?id=7754.

[13] R. Wulf, K. Hinko, and N. Finkelstein, Comparing mechanistic reasoning in open and guided inquiry physics activities, AIP Conf. Proc. 2013, 369 (2013).

[14] L. M. Mayhew and N. D. Finkelstein, Learning to communicate about science in everyday language through informal science education, AIP Conf. Proc. 1179, 205 (2009).

[15] K. Hinko and N. D. Finkelstein, Impacting university physics students through participation in informal science, AIP Conf. Proc. 1513, 178 (2012).

[16] K. A. Hinko, P. Madigan, E. Miller, and N. D. Finkelstein, Characterizing pedagogical practices of university physics students in informal learning environments, Phys. Rev. Phys. Educ. Res. 12, 010111 (2016).

[17] M. B. Bennett, B. Fiedler, and N. D. Finkelstein, Refining a model for understanding and characterizing instructor pedagogy in informal physics learning environments, Phys. Rev. Phys. Educ. Res. 16, 020137 (2020).

[18] K. Hinko, J. Seneca, and N. Finkelstein, Use of scientific language by university physics students communicating to the public, AIP Conf. Proc. 2014, 115 (2014).

[19] C. Thorley, Physicists and outreach: Implications of schools physics outreach programmes from the perspective of the participating physicists, Ph.D. thesis, University College London, 2016.
[20] C. Fracchiolla, B. Prefontaine, and K. Hinko, Community of practice approach for understanding identity development within informal physics programs, Phys. Rev. Phys. Educ. Res. 16, 020115 (2020).

[21] J. D. Adams and P. Gupta, Informal science institutions and learning to teach: An examination of identity, agency, and affordances, J. Res. Sci. Teach. 54, 121 (2017).

[22] C. Fracchiolla, B. Prefontaine, M. Vasquez, and K. Hinko, Is participation in public engagement an integral part of shaping physics students' identity?, Research and Innovation in Physics Education: Two Sides of the Same Coin (Springer, New York, 2020), pp. 225-238.

[23] B. Prefontaine, C. Fracchiolla, M. Vasquez, and K. Hinko, Intense outreach: Experiences shifting university students' identities, in Proceedings of the 2018 Physics Education Research Conference, Washington, DC (AIP, New York, 2018).

[24] C. Fracchiolla, S. Hyater-Adams, N. Finkelstein, and K. Hinko, University physics students' motivations and experiences in informal physics programs, in Proceedings of the 2016 Physics Education Research Conference, Sacramento, CA (AIP, New York, 2016), pp. 124-127.

[25] D. Izadi, J. Willison, K. Hinko, and C. Fracchiolla, Developing an organizational framework for informal physics programs, in Proceedings of the 2019 Physics Education Research Conference, Provo, UT (AIP, New York, 2019), pp. 251-256.

[26] D. Izadi, J. Willison, N. Finkelstein, C. Fracchiolla, and K. Hinko, Mapping the landscape of informal physics educational activities, Phys. Rev. Phys. Educ. Res. (to be published).

[27] C. Lusthaus, G. Anderson, and E. Murphy, Institutional Assessment: A Framework for Strengthening Organizational Capacity for IDRC's Research Partners (IDRC, Ottowa, Canada, 1995).

[28] A. Sodiq and I. Abbott, Reimagining academic staff governors' role in further education college governance, Research in Post-Compulsory Education 23, 138 (2018).

[29] C. Fracchiolla, N. Finkelstein, and K. Hinko, Characterizing models of informal physics programs, in Proceedings of the 2018 Physics Education Research Conference, Washington, DC (AIP, New York, 2018).

[30] R. Wulf, K. Hinko, and N. Finkelstein, Promoting children's agency and communication skills in an informal science program, AIP Conf. Proc. 1513, 430 (2012).

[31] N. Finkelstein, Learning physics in context: A study of student learning about electricity and magnetism, Int. J. Sci. Educ. 27, 1187 (2005). 